\title{
ANALYSIS OF TERAHERTZ DETECTION WITH A 2D HOT-ELECTRON QUANTUM WELL DETECTOR
}

\author{
Y. Huo and G. W. Taylor \\ Electrical and Computer Engineering Department \\ University of Connecticut
}

Storrs, CT 06269

Received 31 October 2002

ABSTRACT: The operation of a previously proposed terahertz $(\mathrm{THz})$ detector is formulated in detail. The detector is based on the hot-electron effect of the $2 D$ electron gas (2DEG) in the quantum well ( $Q W)$ of a GaAs/AlGaAs heterostructure. The interaction between the $\mathrm{THz}$ radiation and the 2DEG, the current enhancement due to hot-electron effect, and the noise performance of the detector are analyzed. (1) 2003 Wiley Periodicals, Inc. Microwave Opt Technol Lett 37: 250-255, 2003; Published online in Wiley InterScience (www.interscience.wiley.com). DOI 10.1002/ mop. 10885

Key words: $\mathrm{THz}$ detection; planar antenna; balance equations; temperature fluctuation noise; compound semiconductor device

\section{INTRODUCTION}

The hot electron quantum well device in a GaAs/AlGaAs heterostructure has been proposed for microwave detection at room temperature [1,2]. The detection is based on the interaction between microwave radiation and the $2 \mathrm{D}$ electron gas (2DEG) in the quantum well. In [1,2], the studied sample was grown by MBE on semi-insulating GaAs substrate and consists of 20 periods of 4.5-nm-thick GaAs QWs doped $n=10^{17} \mathrm{~cm}^{-3}$ and $30-\mathrm{nm}$ undoped $\mathrm{Al}_{0.3} \mathrm{Ga}_{0.7} \mathrm{As}$ barriers. The $\mathrm{MQW}$ is sandwiched between $n=10^{18} \mathrm{~cm}^{-3} \mathrm{GaAs}$ layers to which contacts were provided. The detector was tested at $10 \mathrm{GHz}$ using a waveguide to couple microwave energy to the sample. It was found that, as long as there is a non-zero component of the electric field lying in the layer plane, thermionic current is enhanced due to the radiation. $\mathrm{Al}$ though X-band microwave was used in the preliminary experiments, it was suggested that radiation could be detected up to 10 $\mathrm{THz}$.

The basic setup for the above-mentioned experiment is shown in Figure 1(a). This setup is for experiment only. Due to the small cross-sectional area, very large power density is needed (350 $\mathrm{W} / \mathrm{cm}^{-2}$ ) to couple to the device. In recent years, the integration of detectors with planar antennas and quasi-optical lenses have proved to be appropriate for $\mathrm{THz}$ detection in practical conditions $[3,4]$. Therefore, in this paper we propose to use the setup in Figure 1(b) for the hot-electron detector. The incoming radiation is coupled to the back of the lens and collected by the lens-antenna system to form a stronger field at the device (the polarization of electric field is along the $x$ direction in the QW layer). Although a planar bow-tie antenna is shown in the figure, the planar logperiodic antenna has been shown [5] to be a better candidate, due to its radiation pattern and broad bandwidth.

A theory for the interaction mechanism between the radiation and 2DEG was given in [6]. It considered the power transfer to the 2DEG due to the electron movements in both vertical and in-plane directions, corresponding to $z$ and $x$ directions respectively in Figure 1(b). In section 2, we simplify the theoretical formulation by considering the power transfer from the radiation to $2 D E G$ due to electron movement in the $x$ direction only. The transport in the $z$ direction can be considered as a means of energy dissipation. The analysis is based on balance equations in the 2DEG [7]. The 
relatively wide and the frequency is also relatively high, the distributed nature of the gate of transistor takes care of it. Thus, the proposed model is useful in RF. If gate resistance becomes comparable to the inverse of channel transconductance $g_{m}$, the proposed model becomes essential for designing SOI MOS integrated circuits.

\section{REFERENCES}

I. J.P. Colinge, Silicon-on-Insulator Technology, Materials to VLSI, $2^{\text {mu }}$ Ed., 2002.

2. J.A. Seeger, Microwave theory, components, and devices, Prentice Hall, Englewood Cliffs, NJ (1986).

3. Y.A. El-mansy and A.R. Boothroyd. A simple two dimensional model for IGFET operation in the saturation region, IEEE Trans Electron Devices, ED-24 (1977), 254-262.

4. M. Miura-Mattaush, Analytical MOSFET model for quarter micron technologies, IEEE Trans Computer-Aided Design 3 (1994), 610615.

5. R. Sung, P. Bendix, and M.B. Das, Extraction of high frequency equivalent circuit parameters of submicron gate-length MOSFET's, IEEE Trans Electron Devices 45 (1998), 1769-1775.

6. S.H.M. Jen, C.C. Enz, D.R. Pehlke, M. Schroter, and B.J. Shen, Accurate modeling and parameter extraction for MOS transistors valid upto $10 \mathrm{GHz}$, IEEE Trans Electron Devices 46 (1999), 22172227.

7. G. Dambrine, A. Cappy, F. Heliodore, and E. Playez, A new method for determining the FET small-signal equivalent circuit, IEEE Trans Microwave Theory Techniques 36 (1988), 1151-1159.

8. S. Liu and L.W. Nagel, Small signal MOSFET models for analog circuit design, IEEE J Solid-State Circ SC-17 (1982), 983-998.

9. C. Turchetti, G. Masetti, and Y. Tsidivis, On the small signal behaviour of the MOS transistor in quasi-state operation, Solid-State Electron 26 (1983), 941-949.

10. E.A.A. Allam and T. Manku, A small-signal MOSFET model for radio frequency IC applications, IEEE Trans Computer-Aided Design 16 (1997), 437-447.

11. A. Goswami, A. Agrawal, C.T. Thuruthiyil, M. Gupta, and R.S. Gupta, A small-signal analytical MOSFET model for microwave frequency applications, Microwave Optical Technol Lett 25 (2000), 346-352.

12. J.P. Raskin, R. Gilon, J. Chen, D.V. Janvier, and J.P. Colinge, Accurate SOI MOSFET characterization at microwave frequencies for device performance optimization and analog modeling, IEEE Trans Electron Devices 45 (1998), 1017-1025.

13. C.L. Chen et al., High frequency characterization of sub- $0.25-\mu \mathrm{m}$ fully depleted silicon-on-insulator MOSFETs, IEEE Electron Device Lett 21 (2000), 497-499. 